\section{BMJ Open \\ Respiratory \\ Research}

To cite: Davidson AC, Banham S, Elliott M, et al. British Thoracic Society/ Intensive Care Society Guideline for the ventilatory management of acute hypercapnic respiratory failure in adults. BMJ Open Resp Res 2016;3:e000133. doi:10.1136/bmjresp-2016000133

\section{- Additional material is available. To view please visit the journal (http://dx.doi.org/ 10.1136/bmjresp-2016- 000133) \\ Healthcare providers need to use clinical judgement, knowledge and expertise when deciding whether it is appropriate to apply recommendations for the management of patients. The recommendations cited are a guide and may not be appropriate for use in all situations. The guidance provided does not override the responsibility of healthcare professionals to make decisions appropriate to the circumstances of each patient, in consultation with the patient and/or their guardian or carer.}

Received 18 February 2016 Accepted 28 February 2016

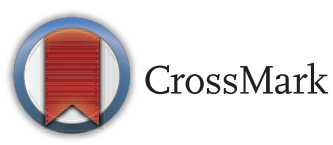

For numbered affiliations see end of article.

Correspondence to Dr Craig Davidson; craigdavidson@doctors.org.uk

\title{
British Thoracic Society/Intensive Care Society Guideline for the ventilatory management of acute hypercapnic respiratory failure in adults
}

Craig Davidson, ${ }^{1}$ Steve Banham, ${ }^{2}$ Mark Elliott, ${ }^{3}$ Daniel Kennedy, ${ }^{4}$ Colin Gelder, ${ }^{5}$ Alastair Glossop, ${ }^{6}$ Colin Church, ${ }^{7}$ Ben Creagh-Brown, ${ }^{8}$ James Dodd, ${ }^{9}$ Tim Felton, ${ }^{10}$ Bernard Foëx, ${ }^{11}$ Leigh Mansfield, ${ }^{12}$ Lynn McDonnell, ${ }^{13}$ Robert Parker, ${ }^{14}$ Caroline Patterson, ${ }^{15}$ Milind Sovani, ${ }^{16}$ Lynn Thomas, ${ }^{17}$ BTS Standards of Care Committee Member, British Thoracic Society/Intensive Care Society Acute Hypercapnic Respiratory Failure Guideline Development Group, On behalf of the British Thoracic Society Standards of Care Committee

\section{INTRODUCTION}

The British Thoracic Society (BTS) published the guideline 'The use of non-invasive ventilation in acute respiratory failure' in 2002. ${ }^{1}$ This was in response to trials that had demonstrated that non-invasive ventilation (NIV) was an alternative to invasive mechanical ventilation (IMV) in life-threatening respiratory acidosis due to acute exacerbations of chronic obstructive pulmonary disease (AECOPD). It drew attention to evidence that, when NIV was used in the less severely unwell patient, it also limited progression to more severe respiratory failure. ${ }^{2}$ The trial also demonstrated the feasibility, of delivering NIV on general medical or admission wards that had enhanced support and when staff were provided with ongoing training.

In subsequent years, NIV has been shown to deliver better rather than equivalent outcomes to invasive ventilation in AECOPD and better evidence has accumulated for the use of NIV in non-COPD disease in the intervening years. Repeated national audits have, however, raised concerns that expected patient benefit is not being delivered and have pointed to a number of process deficiencies. $^{3-5}$ There is also the risk, in the absence of justifying trial evidence, that the preferred use of NIV in AECOPD might be extended to all hypercapnic patients, irrespective of circumstance or underlying disease process. That this is a real risk might be inferred from the BTS audits where the indication for NIV was not COPD in over $30 \%$ of cases. ${ }^{34}$

NIV development in the UK has been largely outside the organisational 'umbrella' of critical care. This may have adversely affected resource allocation and contributed to a lack of integration in NIV and IMV patient pathways. Other unintended consequences might be a restriction on access to invasive ventilation and delay in the development of extended applications of NIV, such as accelerating extubation and its use in the management of post-extubation respiratory failure, in intensive care units. ${ }^{6}$ Intensive care specialists may have little experience of the ability of domiciliary NIV to reverse chronic cardio-respiratory failure and this may lead to underestimating survival, particularly in advanced neuromuscular disease (NMD) or chest wall disease (CWD).

For these varied reasons, the need for up to date guidance was acknowledged by BTS and the Intensive Care Society (ICS). The aim of the guideline is to draw attention to the evidence of suboptimal care in acute hypercapnic respiratory failure (AHRF) in the UK, provide an overview of the evidence supporting the use of invasive and NIV, encourage better communication between admitting clinicians and critical care services and the creation of AHRF patient pathways and improve resourcing, training, outcomes and patient experience for all adults who develop acute hypercapnic respiratory failure.

Conventionally, a $\mathrm{pH}<7.35$ and a PCO2 $>6.5 \mathrm{kPa}$ defines acute respiratory failure and, when persisting after initial medical therapy, have been used as threshold values for considering the use of NIV. More severe degrees of acidosis, such as $\mathrm{pH}<7.25$, 
have been used as a threshold for considering provision of IMV. AHRF results from an inability of the respiratory pump, in concert with the lungs, to provide sufficient alveolar ventilation to maintain a normal arterial PCO2. Coexistent hypoxaemia is usually mild and easily corrected.

AHRF complicates around $20 \%$ of acute exacerbations of COPD. ${ }^{2}{ }^{7}$ It signals advanced disease, a high risk of future hospitalisations and limited long-term prognosis. The median survival following recovery from AHRF was 1 year in a large case series. ${ }^{7}$ Around $12 \%$ of hypercapnic COPD patients died during the index admission and this increased to $33 \%$ if the respiratory acidosis developed after hospitalisation. In asthma, acute hypercapnia also signals an increased risk of death and an increased likelihood of future life-threatening attacks. ${ }^{8}$ The same risks apply to AHRF complicating cystic fibrosis and bronchiectasis although this has not been formally reported. In the neuromuscular and CWD, including morbid obesity, respiratory pump failure is often insidious in its onset but AHRF may be acute and unexpected. Acute on chronic 'decompensated' episodes of AHRF are more common and normally indicate the future need for domiciliary NIV.

The guideline is targetted at medical, nursing and physiotherapy staff working in emergency receiving rooms, medical assessment units, admission wards, respiratory wards and in high dependency and critical care units. The Guideline applies to adults and does not cover the management of AHRF due to cardiac failure, trauma or acute brain injury. It refers to domiciliary NIV but does not aim to provide guidance on this. The use of non-invasive ventilation is intentionally more extensively covered than IMV because the evidence and the clinical experience of NIV is more recent and because the technical aspects of IMV are covered by standard texts.

The guideline does not cover the management of AHRF due to cardiac failure, trauma or acute brain injury. The guideline refers to domiciliary NIV but does not aim to provide guidance on this. The use of noninvasive ventilation is more extensively covered than IMV because the evidence and the clinical experience in its use is more recent and because the technical aspects concerning IMV are well covered by standard texts.

\section{Methodology}

Clinical questions were gathered in the PICOT (Patient, Intervention, Comparison, Outcome and Time) format to define the scope of the guideline and inform the literature search. Systematic electronic database searches were conducted in order to identify potentially relevant studies for inclusion in the guideline. For each clinical question the following databases were searched: Ovid MEDLINE (including MEDLINE In-Process), Ovid EMBASE, EMSCO CINAHL, Ovid PsycINFO and the
Cochrane Library (including the Cochrane Database of Systematic Reviews, the Database of Abstracts of Reviews of Effects and the Cochrane Central Register of Controlled Trials). An initial search was carried out in November 2010 using a combination of indexed and free-text terms defining the clinical questions that had been agreed by the guideline group as important in formulating guidelines in AHRF. It was limited to studies after 1990, in adults, in journals published in English and where at least an abstract was available. The searches identified a total of 582 potential papers which were subsequently supplemented by publications known to members or resulting from additional searches undertaken by the writing groups after 2010. The literature search was run again for relevant publications between 2010 and 2013 in September 2013, yielding a further 308 potentially relevant references. Additional references were subsequently included from personal collections.

Appraisal was performed using the criteria stipulated by the AGREE collaboration. Each paper was appraised by at least two reviewers. The writing lead for each section read the title and abstract of papers identified and agreed with at least one member of each writing group whether such a paper was definitely, possibly relevant or not relevant to the section. The criteria used were whether the paper addressed a clinical question, whether the study method used was satisfactory and that it was available in English. Full papers were obtained for all relevant or possibly relevant abstracts. Two members for each section independently appraised each paper using the SIGN critical appraisal checklists. An evidence level was assigned to each study using SIGN methodology (table 1) and recommendations formulated according to this evidence base (table 2).

\section{Table 1 SIGN levels of evidence}

$1++$ High-quality meta-analyses, systematic reviews of RCTs, or RCTs with a very low risk of bias.

1+ Well-conducted meta-analyses, systematic reviews, or RCTs with a low risk of bias.

1- Meta-analyses, systematic reviews, or RCTs with a high risk of bias.

$2++$ High-quality systematic reviews of case control or cohort or studies High-quality case-control or cohort studies with a very low risk of confounding or bias and a high probability that the relationship is causal.

2+ Well-conducted case control or cohort studies with a low risk of confounding or bias and a moderate probability that the relationship is causal.

2- Case control or cohort studies with a high risk of confounding or bias and a significant risk that the relationship is not causal.

3 Non-analytic studies, for example, case reports, case series.

4 Expert opinion.

$\mathrm{RCT}$, randomised controlled trial. 


\section{Table 2 SIGN grades of recommendations}

A At least one meta-analysis, systematic review, or RCT rated as $1++$, and directly applicable to the target population; or

A body of evidence consisting principally of studies rated as $1+$, directly applicable to the target population, and demonstrating overall consistency of results.

B A body of evidence including studies rated as 2++, directly applicable to the target population, and demonstrating overall consistency of results; or Extrapolated evidence from studies rated as $1++$ or $1+$

C A body of evidence including studies rated as 2+, directly applicable to the target population and demonstrating overall consistency of results; or Extrapolated evidence from studies rated as 2++.

D Evidence level 3 or 4; or Extrapolated evidence from studies rated as $2+$.

$\mathrm{RCT}$, randomised controlled trial.

Recommendations were graded from A to D, using the SIGN Grading System (table 2), as indicated by the strength of the evidence as listed in the tables. Important practical points that lack research evidence were highlighted as 'Good Practice Points'.

Good practice points

Recommended best practice based on the clinical experience of the guideline development group.

\section{Units}

Intrathoracic pressure and pressures relating to mechanical ventilation are presented as cm H2O. Arterial blood gas tensions are presented as $\mathrm{kPa}$.

\section{Summary of recommendations and good practice points}

A summary of recommendations and good practice points from the full guideline document ${ }^{9}$ are included below.

For the detailed review of evidence and the full bibliography please refer to the full guideline and the information available on the BTS website at: https://www. brit-thoracic.org.uk/guidelines-and-quality-standards/

All figures 1-3 and table 3 and boxes 1-4 from the full Guideline are reproduced for reference.

\section{SUMMARY OF RECOMIMENDATIONS}

Principles of mechanical ventilation

Modes of mechanical ventilation

\section{Recommendation}

1. Pressure targeted ventilators are the devices of choice for acute NIV (grade B).

Good practice points

- Pressure support and pressure control modes are effective.

- Only ventilators designed specifically to deliver NIV should be used.
Choice of interface for NIV

\section{Recommendation}

2. A full face mask should usually be the first type of interface used (grade D).

Good practice points

- A range of masks and sizes is required and staff involved in delivering NIV need training in and experience of using them.

- NIV circuits must allow adequate clearance of exhaled air through an exhalation valve or an integral exhalation port on the mask.

\section{Indications for and contraindications to NIV in AHRF \\ Recommendation}

3. The presence of adverse features increase the risk of NIV failure and should prompt consideration of placement in HDU/ICU (grade C) (see figure 1).

Good practice points

- Adverse features should not, on their own, lead to withholding a trial of NIV.

- The presence of relative contra-indications necessitates a higher level of supervision, consideration of placement in HDU/ICU and an early appraisal of whether to continue NIV or to convert to IMV.

\section{Monitoring during NIV}

Good practice points

- Oxygen saturation should be continuously monitored.

- Intermittent measurement of pCO2 and $\mathrm{pH}$ is required.

- ECG monitoring is advised if the patient has a pulse rate $>120 \mathrm{bpm}$ or if there is dysrhythmia or possible cardiomyopathy.

\section{Supplemental oxygen therapy with NIV}

\section{Recommendations}

4. Oxygen enrichment should be adjusted to achieve $\mathrm{SaO} 288-92 \%$ in all causes of AHRF treated by NIV (grade A).

5. Oxygen should be entrained as close to the patient as possible (grade $\mathrm{C}$ ).

Good practice points

- As gas exchange will improve with increased alveolar ventilation, NIV settings should be optimised before increasing the $\mathrm{FiO} 2$.

- The flow rate of supplemental oxygen may need to be increased when ventilatory pressure is increased to maintain the same $\mathrm{SaO} 2$ target.

- Mask leak and delayed triggering may be caused by oxygen flow rates $>4 \mathrm{~L} / \mathrm{min}$ which risks promoting or exacerbating patient-ventilator asynchrony. The requirement for high flow rates should prompt a careful check for patient-ventilator asynchrony.

- A ventilator with an integral oxygen blender is recommended if oxygen at $4 \mathrm{~L} / \mathrm{min}$ fails to maintain $\mathrm{SaO} 2$ $>88 \%$. 


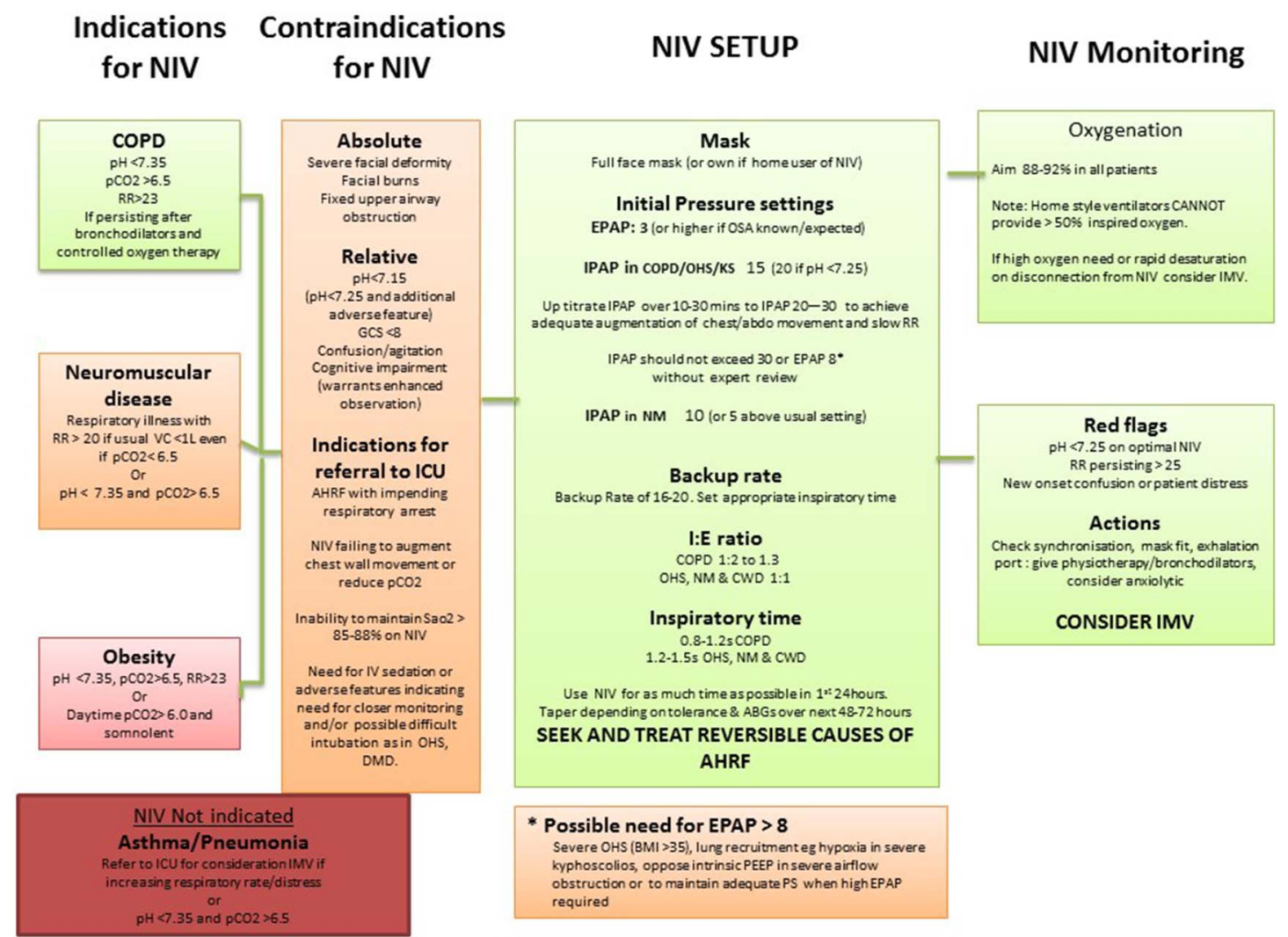

Figure 1 Summary for providing acute non-invasive ventilation (NIV).

\section{Humidification with NIV}

\section{Recommendation}

6. Humidification is not routinely required (grade D). Good practice point

Heated humidification should be considered if the patient complains of mucosal dryness or if respiratory secretions are thick and tenacious.

\section{Bronchodilator therapy with NIV}

\section{Good practice points}

- Nebulised drugs should normally be administered during breaks from NIV.

- If the patient is dependent on NIV, bronchodilator drugs can be given via a nebuliser inserted into the ventilator tubing.

Figure 2 Guide to initial settings and aims with IMV. IMV, invasive mechanical ventilation.

\section{Sedation with NIV}

\section{Recommendations}

7. Sedation should only be used with close monitoring (grade D).

8. Infused sedative/anxiolytic drugs should only be used in an HDU or ICU setting (grade D).

9. If intubation is not intended should NIV fail, then sedation/anxiolysis is indicated for symptom control in the distressed or agitated patient (grade D).

Good practice point

In the agitated/distressed and/or tachypneic individual on NIV, intravenous morphine $2.5-5 \mathrm{mg}$ ( \pm benzodiazepine) will provide symptom relief and may improve tolerance of NIV. 


\begin{tabular}{|c|c|c|}
\hline $\begin{array}{l}\text { Immediate Clinical } \\
\text { Assessment } \\
\text { Oxygenation target 88-92\% } \\
\text { Acid-Base Status? } \\
\text { Evidence of other organ } \\
\text { dysfunction? } \\
\text { Co-morbidities? } \\
\text { Administer steroids, } \\
\text { bronchodilators, antibiotics as } \\
\text { indicated and get specialist } \\
\text { therapy help for NM/OHS } \\
\text { patients. } \\
\text { Consider predisposition to } \\
\text { AHRF [link with HMV team] } \\
\text { Enquire about advanced care } \\
\text { plans (confirm or commence } \\
\text { discussion) }\end{array}$ & $\begin{array}{l}\text { Assisted Ventilation Plan } \\
\text { Options: } \\
\text { Intubation and transfer to ICU for IMV } \\
\text { NIV with transfer to ICU as risk of requiring } \\
\text { IMV } \\
\text { NIV before/after transfer to NIV unit } \\
\text { NIV before/after transfer to acute ward } \\
\text { with specialist support } \\
\text { Non implementation or discontinuation of } \\
\text { assisted ventilation } \\
\text { Review patient and family wishes } \\
\text { Ensure NIV experienced*clinical input and } \\
\text { assistance of ICU if needed } \\
\text { Use locally agreed protocols for AHRF } \\
\text { management } \\
\text { Ensure frequent review of progress and } \\
\text { agreed avenues for escalation or de- } \\
\text { escalation } \\
\text { Document care plans and audit outcomes } \\
\text { *A NIV experienced clinician will have undergone } \\
\text { specific training and be able to demonstrate } \\
\text { possession of all of the appropriate competencies. }\end{array}$ & $\begin{array}{l}\text { Recovery and discharge } \\
\text { phase } \\
\text { Review reasons/routeof admission and } \\
\text { consider methods to improveif these } \\
\text { were problematic } \\
\text { Discuss future care planning with } \\
\text { patient/family and inform community } \\
\text { services of the result of such discussion. } \\
\text { Arrange early specialist review, } \\
\text { pulmonary rehabilitation \& help with } \\
\text { smoking cessation as indicated } \\
\text { Provide warning card/inform } \\
\text { ambulance services re future need for } \\
\text { controlled oxygen therapy } \\
\text { Consider referral to home NIV service } \\
\text { eg NMD cases or suspected sleep } \\
\text { disordered breathing } \\
\text { Learn from any identified mistakes } \\
\text { through multi professional review. }\end{array}$ \\
\hline
\end{tabular}

Figure 3 The three phases of patient management in AHRF.

\section{NIV complications}

\section{Good practice points}

- Minor complications are common but those of a serious nature are rare. Patients should be frequently assessed to identify potential complications of NIV.

- Care is needed to avoid overtightening of masks.

- Previous episodes of ventilator-associated pneumothorax warrant consideration of admission to HDU/ ICU and use of NIV at lower than normal inspiratory pressures.

- The development of a pneumothorax usually requires intercostal drainage and review of whether to continue with NIV.

\section{Sputum retention}

\section{Recommendations}

10. In patients with NMD, mechanical insufflation and exsufflation should be used, in addition to standard physiotherapy techniques, when cough is ineffective and there is sputum retention (grade B).

11. Minitracheostomy may have a role in aiding secretion clearance in cases of weak cough (NMD/CWD) or excessive amounts (COPD, CF) (grade D).

\section{Modes of IMV}

\section{Recommendations}

12. Spontaneous breathing should be established as soon as possible in all causes of AHRF (grade C).

13. Controlled IMV may need to be continued in some patients due to severe airflow obstruction, weak muscles leading to poor triggering or to correct chronic hypercapnia (grade C).

Good practice point

In the obstructive diseases, controlled IMV should be continued until airway resistance falls.

\section{Table 3 Technical issues: a guide for when NIV is failing}

\begin{tabular}{|c|c|c|}
\hline Problem & Cause(s) & Solution (s) \\
\hline $\begin{array}{l}\text { Ventilator cycling independently of } \\
\text { patient effort }\end{array}$ & $\begin{array}{l}\text { Inspiratory trigger sensitivity is too high } \\
\text { Excessive mask leak }\end{array}$ & $\begin{array}{l}\text { Adjust trigger } \\
\text { Reduce mask leak }\end{array}$ \\
\hline Ventilator not triggering despite visible & Excessive mask leak & Reduce mask leak \\
\hline patient effort & Inspiratory trigger sensitivity is too low & $\begin{array}{l}\text { Adjust trigger } \\
\text { For NM patients consider switch to PCV }\end{array}$ \\
\hline $\begin{array}{l}\text { Inadequate chest expansion despite } \\
\text { apparent triggering }\end{array}$ & Inadequate Tidal volume & $\begin{array}{l}\text { Increase IPAP. In NM or chest wall } \\
\text { disease consider longer } \mathrm{Ti}\end{array}$ \\
\hline Chest/abdominal paradox & Upper airway obstruction & $\begin{array}{l}\text { Avoid neck flexion } \\
\text { Increase EPAP }\end{array}$ \\
\hline Premature expiratory effort by patient & Excessive Ti or IPAP & Adjust as necessary \\
\hline
\end{tabular}


Box 1 Indications for invasive mechanical ventilation in acute exacerbations of chronic obstructive pulmonary disease
Imminent respiratory arrest
- Severe respiratory distress
- Failure of or contraindications to non-invasive ventilation (NIV)
- Persisting $\mathrm{pH}<7.15$ or deterioration in $\mathrm{pH}$ despite NIV
Depressed consciousness (GCS $<8$ )

\section{Invasive ventilation strategy}

\section{Recommendations}

14. During controlled ventilation, dynamic hyperinflation should be minimised by prolonging expiratory time (I:E ratio $1: 3$ or greater) and setting a low frequency (10-15 breaths per minute) (grade C) (see figure 2).

15. Permissive hypercapnia (aiming for $\mathrm{pH}$ 7.2-7.25) may be required to avoid high airway pressures when airflow obstruction is severe (grade $\mathrm{D}$ ).

16. Carbonic anhydrase inhibitors should not be routinely used in AHRF (grade C).

\section{Positive end expiratory pressure}

\section{Recommendation}

17. Applied extrinsic positive end expiratory pressure (ePEEP) should not normally exceed $12 \mathrm{~cm}$ (grade $\mathrm{C}$ ) .

\section{Sedation in IMV}

\section{Recommendation}

18. Sedation should be titrated to a specific level of alertness (grade B).

\section{Patient-ventilator asynchrony}

\section{Recommendations}

19. Ventilator asynchrony should be considered in all agitated patients (including NIV) (grade C).

20. As patients recover from AHRF, ventilator requirements change and ventilator settings should be reviewed regularly (grade $\mathrm{C}$ ).

\section{The use and timing of a tracheostomy \\ Recommendations}

21. Performing routine tracheostomy within 7 days of initiating IMV is not recommended (grade A).

Box 2 Risk factors for extubation failure following invasive mechanical ventilation (IMV)

Positive fluid balance

Raised rapid shallow breathing index during SBT

Pneumonia or pulmonary disease as the cause requiring IMV

Increased age

Prolonged duration of IMV

Anaemia

Increased severity of illness

Low albumin

Previous failed extubation

Bulbar dysfunction
Box 3 Essential requirements for a non-invasive ventilation (NIV) service

Specifically identified area(s) for NIV treatment at level 2 equivalence.

- Staffing levels above that of a general medical ward with one nurse for every 2 NIV cases (especially during the first $24 \mathrm{~h}$ of treatment).

- Locally developed NIV protocols (based on published best practice guides) uniformly applied across all areas

- A designated lead with a 'core' multidisciplinary group (physicians, nurses, physiotherapists) co-ordinating hospital wide NIV service provision and performance

- Access to expert support for NIV technical advice in and out of hours

- Mechanisms for regular audit

- Regular staff educational updates and training module for new staff

22. The need for and timing of a tracheostomy should be individualised (grade $\mathrm{D}$ ).

Good practice points

- In AHRF due to COPD, and in many patients with NMD or obesity hypoventilation syndrome, NIV supported extubation should be employed in preference to inserting a tracheostomy.

- In AHRF due to NMD, alongside discussion with the patient and carers, the decision to perform tracheostomy should be multidisciplinary and should involve discussion with a home ventilation unit.

\section{Management of hypercapnic respiratory failure}

Prevention of AHRF in AECOPD

\section{Recommendations}

23. In AHRF due to AECOPD, controlled oxygen therapy should be used to achieve target saturations of $88-92 \%$ (grade A).

Good practice point

Controlled oxygen therapy should be used to achieve a target saturation of $88-92 \%$ in ALL causes of AHRF.

\section{Box 4 Discharge checklist after an episode of AHRF}

- Arrange early specialist review, pulmonary rehabilitation and help with smoking cessation as indicated.

- Consider early home visit for example, outreach chronic obstructive pulmonary disease team/community nurses

- Discuss future care planning with patient/family and inform community services of the result of such discussion.

- Provide warning card/inform ambulance services re future need for controlled oxygen therapy

- Consider referral to home non-invasive ventilation service for example, neuromuscular disease cases or suspected sleepdisordered breathing

- Review reasons/route of admission and consider methods to improve if these were problematic

- Learn from any identified mistakes through multi professional review. 
Role of NIV in AECOPD

\section{Recommendations}

24. For most patients with AECOPD, the initial management should be optimal medical therapy and targeting an oxygen saturation of $88-92 \%$ (grade A).

25. NIV should be started when $\mathrm{pH}<7.35$ and $\mathrm{pCO} 2$ $>6.5 \mathrm{kPa}$ persist or develop despite optimal medical therapy (grade A).

26. Severe acidosis alone does not preclude a trial of NIV in an appropriate area with ready access to staff who can perform safe endotracheal intubation (grade B).

27. The use of NIV should not delay escalation to IMV when this is more appropriate (grade $\mathrm{C}$ ).

28. The practice of NIV should be regularly audited to maintain standards (grade $\mathrm{C}$ ).

\section{Starting NIV in COPD}

Good practice points

- Arterial blood gas measurement is needed prior to and following starting NIV.

- Chest radiography is recommended but should not delay initiation of NIV in severe acidosis.

- Reversible causes for respiratory failure should be sought and treated appropriately.

- At the start of treatment, an individualised patient plan (involving the patient wherever possible) should document agreed measures to be taken in the event of NIV failure.

\section{Prognostic features relating to use of NIV in COPD}

\section{Recommendations}

29. Advanced age alone should not preclude a trial of NIV (grade A).

30. Worsening physiological parameters, particularly $\mathrm{pH}$ and respiratory rate, indicate the need to change the management strategy. This includes clinical review, change of interface, adjustment of ventilator settings and considering proceeding to endotracheal intubation (grade A).

Good practice point

If sleep-disordered breathing predates AHRF, or evidence of it complicates an episode, the use of a controlled mode of NIV overnight is recommended.

\section{Duration of NIV in COPD}

\section{Recommendation}

31. NIV can be discontinued when there has been normalisation of $\mathrm{pH}$ and $\mathrm{pCO} 2$ and a general improvement in the patients condition (grade B).

Good practice points

- Time on NIV should be maximised in the first $24 \mathrm{~h}$ depending on patient tolerance and/or complications.

- NIV use during the day can be tapered in the following 2-3 days, depending on pCO2 self-ventilating, before being discontinued overnight.
Optimising NIV delivery and technical considerations

Good practice point

Before considering NIV to have failed, always check that common technical issues have been addressed and ventilator settings are optimal (see table 3).

\section{Indications for IMV in AECOPD}

\section{Recommendations}

32. IMV should be considered if there is persistent or deteriorating acidosis despite attempts to optimise delivery of NIV (grade A).

33. Intubation should be performed in respiratory arrest or periarrest unless there is rapid recovery from manual ventilation/provision of NIV (grade D).

34. Intubation is indicated in management of AHRF when it is impossible to fit/use a non-invasive interface for example, severe facial deformity, fixed upper airway obstruction, facial burns (grade $\mathrm{D}$ ).

35. Intubation is indicated where risk/benefit analysis by an experienced clinician favours a better outcome with IMV than with NIV (grade D).

\section{Outcome following NIV or IMV in AECOPD \\ Recommendations}

36. Prognostic tools may be helpful to inform discussion regarding prognosis and with regard to the appropriateness of IMV, but with the caveat that such tools are poorly predictive for individual patient use (grade B).

37. Clinicians should be aware that they are likely to underestimate survival in AECOPD treated by IMV (grade $\mathrm{B}$ ).

38. Clinicians should discuss management of possible future episodes of AHRF with patients, following an epsiode requiring ventilatory support, because there is a high risk of recurrence (grade B).

\section{Acute asthma}

\section{Recommendations}

39. NIV should not be used in patients with acute asthma exacerbations and AHRF (grade $\mathrm{C}$ ).

40. Acute (or acute on chronic) episodes of hypercapnia may complicate chronic asthma. This condition closely resembles COPD and should be managed as such (grade D).

\section{Non-CF bronchiectasis Recommendations}

41. In patients with non-CF bronchiectasis and AHRF, controlled oxygen therapy should be used (grade D).

42. In patients with non-CF bronchiectasis, NIV should be started in AHRF using the same criteria as in AECOPD (grade B).

43. In patients with non-CF bronchiectasis, NIV should usually be tried before resorting to IMV in those with less severe physiological disturbance (grade $\mathrm{C}$ ).

44. In non-CF bronchiectasis, the patient's clinical condition prior to the episode of AHRF, and the reason for 
the acute deterioration, should be evaluated and used to inform the decision about providing IMV (grade C).

\section{Good practice points}

- In patients with non-CF bronchiectasis, the precipitating cause is important in determining short-term prognosis.

- Health status prior to the episode of AHRF is an important predictor of outcome.

\section{Cystic fibrosis}

\section{Recommendations}

45. In patients with $\mathrm{CF}$, controlled oxygen therapy should be used in AHRF (grade D).

46. In patients with CF, NIV is the treatment of choice when ventilatory support is needed $(\mathrm{C})$.

47. In patients with CF, specialised physiotherapy is needed to aid sputum clearance (D).

48. In patients with CF, a minitracheostomy combined with NIV may offer greater chance of survival than resorting to IMV (D).

\section{Restrictive lung diseases}

\section{NMD and CWD}

\section{Recommendations}

49. Controlled oxygen therapy should be used in patients with NMD or CWD and AHRF (grade D).

50. NIV should almost always be trialled in the acutely unwell NMD or CWD patient with hypercapnia. Do not wait for acidosis to develop (grade D).

51. In patients with NMD or CWD, NIV should be considered in acute illness when vital capacity (VC) is known to be $<1 \mathrm{~L}$ and $\mathrm{RR}>20$ even if normocapnic (grade D).

52. In patients with NMD or CWD, consider controlled ventilation as triggering may be ineffective (grade $\mathrm{D}$ ).

53. In NMD or CWD, unless escalation to IMV is not desired by the patient, or is deemed to be inappropriate, intubation should not be delayed if NIV is failing (grade D).

Good practice points

- Individuals with NMD and CWD who present with AHRF should not be denied acute NIV.

- NIV is the ventilation mode of choice because patients with NMD or CWD tolerate it well and because extubation from IMV may be difficult.

- In patients with NMD or CWD, deterioration may be rapid or sudden, making HDU/ICU placement for therapy more appropriate.

- In patients with NMD or CWD, senior/experienced input is needed in care planning and is essential if differences in opinion exist or develop between medical staff and patient representatives.

- In patients with NMD, it should be anticipated that bulbar dysfunction and communication difficulties, if present, will make NIV delivery difficult and may make it impossible.

- Discussion about NIV and IMV, and patients, wishes with respect to cardiopulmonary resuscitation, should occur as part of routine care of patients with NMD or CWD.

- In patients with NMD or CWD, nocturnal NIV should usually be continued following an episode of AHRF, pending discussion with a home ventilation service.

\section{NIV failure and discontinuing NIV following recovery in NMD} and CWD

Good practice points

- In patients with NMD or CWD, intolerance of the mask and severe dyspnoea are less likely to cause NIV failure. Bulbar dysfunction makes NIV failure more likely.

- Deterioration in patients with NMD or CWD may be very sudden. Difficulty achieving adequate oxygenation or rapid desaturation during a break from NIV are important warning signs.

- In patients with NMD or CWD, the presence of bulbar dysfunction, more profound hypoxaemia or rapid desaturation during NIV breaks, suggests that placement in HDU/ICU is indicated.

\section{IMV in NMD/CWD}

\section{Recommendations}

54. In patients with NMD or CWD, senior staff should be involved in decision-making, in conjunction with home mechanical ventilation specialists, if experience is limited, and especially when the appropriateness of IMV is questioned (grade D).

55. Advance care planning, particularly around the potential future use of IMV, is recommended in patients with progressive NMD or CWD. This may best be supported by elective referral to a home ventilation service (grade D).

\section{IMV strategy in NMD and CWD}

Good practice points

- Patients with NMD usually require lower levels of pressure support.

- Patients with CWD usually require higher levels of pressure support.

- PEEP in the range 5-10 is commonly required to increase residual volume and reduce oxygen dependency in both patient groups.

\section{Obesity hypoventilation syndrome}

\section{Recommendations}

56. Controlled oxygen therapy should be used in patients with hypoventilation syndrome (OHS) and AHRF (grade D).

57. In patients with OHS, NIV should be started in AHRF using the same criteria as in AECOPD (grade B).

58. NIV is indicated in some hospitalised obese hypercapnic patients with day time somnolence, sleepdisordered breathing and/or right heart failure in the absence of acidosis (grade $\mathrm{D}$ ). 
NIV settings and placement in OHS

Good practice points

- High inspiratory positive airway pressure (IPAP) and expiratory positive airway pressure (EPAP) settings are commonly required in patients with OHS (eg, IPAP $>30$, EPAP $>8$ ).

- Volume control (or volume assured) modes of providing NIV may be more effective when high inflation pressures are required.

\section{NIV failure in OHS}

Good practice points

- Fluid overload commonly contributes to ventilatory failure in patients with OHS, and its degree is easily underestimated.

- Forced diuresis may be useful.

- As the risk of NIV failure is greater, and intubation may be more difficult, placement in HDU/ICU for NIV is recommended.

\section{Discontinuing NIV in OHS}

Good practice points

- NIV can be discontinued as in patients with AECOPD.

- Many patients with AHRF secondary to OHS will require long-term domiciliary support (CPAP or NIV).

- Following an episode of AHRF referral to a home ventilation service is recommended.

\section{IMV strategy in OHS}

Good practice points

- In patients with OHS, pressure controlled MV is recommended initially.

- In patients with OHS, high PEEP settings may be needed to recruit collapsed lung units and correct hypoxaemia.

- In patients with OHS, a forced diuresis is often indicated.

\section{Weaning from IMV \\ Introduction \\ Recommendations}

59. Treating the precipitant cause of AHRF, normalising $\mathrm{pH}$, correcting chronic hypercapnia and addressing fluid overload should all occur before starting weaning (grade D).

60. A brain natriuretic petide (BNP)-directed fluid management strategy should be considered in patients with known left ventricular dysfunction (grade B).

\section{Weaning methods}

\section{Recommendations}

61. Assessment of the readiness for weaning should be undertaken daily (grade C).

62. A switch from controlled to assisted IMV should be made as soon as patient recovery allows (grade $\mathrm{C}$ ).
63. IMV patients should have a documented weaning plan (grade B).

\section{Assessing readiness for discontinuation of mechanical ventilation \\ Recommendation}

64. A 30-min spontaneous breathing trial (SBT) should be used to assess suitability for extubation (grade B).

65. Factors including upper airway patency, bulbar function, sputum load and cough effectiveness should be considered prior to attempted extubation (grade $\mathrm{D}$ ).

\section{Outcome following extubation \\ Recommendation}

66. Care is needed to identify factors that increase the risk of extubation failure so that additional support, such as NIV or cough assist, can be provided (grade B).

\section{Weaning protocols}

\section{Recommendations}

67. Although an organised and systematic approach to weaning is desirable, protocols should be used with caution in patients with AHRF (grade B).

68. The use of computerised weaning cannot be recommended in AHRF (grade D).

\section{The use of NIV in the ICU}

\section{Planned NIV to speed weaning from IMV}

\section{Recommendation}

69. NIV is recommended to aid weaning from IMV in patients with AHRF secondary to COPD (grade B).

70. In other causes of AHRF, NIV may have a role in shortening the duration of IMV when local expertise in its use exists (and of cough assist when indicated) and there are features present that indicate extubation is likely to be successful (grade D).

\section{NIV in high risk patients}

Recommendation

71. Prophylactic use of NIV should be considered to provide post extubation support in patients with identified risk factors for extubation failure (grade B).

\section{NIV as 'rescue' therapy postextubation}

\section{Recommendations}

72. NIV should not be used routinely for unexpected post-extubation respiratory failure (grade B).

73. In COPD, a trial of NIV may be justified for unexpected post-extubation respiratory failure where local expertise exists (grade D).

\section{Care planning and delivery of care}

Appropriate care environments for the delivery of NIV

\section{Recommendations}

74. NIV services should operate under a single clinical lead with formal working links with the ICU (grade D). 
75. The severity of AHRF, and evidence of other organ dysfunction, should influence the choice of care environment (grade $\mathrm{C}$ ).

76. NIV should take place in a clinical environment with enhanced nursing and monitoring facilities that are beyond that of a general medical ward (grade $\mathrm{C}$ ).

77. Initial care plans should include robust arrangements for escalation, anticipating that around $20 \%$ of AHRF cases should be managed in a level 2 or 3 environment (grade $\mathrm{C}$ ).

Good practice points

- A 2-4 bedded designated NIV unit (located within a medical high dependency area or within a respiratory ward with enhanced staffing levels) provides a sound basis for the provision of NIV in a DGH serving a population of 250000 and with an average prevalence of COPD.

- Areas providing NIV should have a process for audit and interdisciplinary communication.

\section{Palliative care and advanced care planning}

\section{Recommendations}

78. Clinicians delivering NIV or IMV should have ready access to palliative medicine (grade $\mathrm{D}$ ).

79. Multidisciplinary advance care planning should be an integral part of the routine outpatient management of progressive or advanced disease and care plans should be reviewed on presentation during an episode of AHRF (grade D).

80. The use of NIV may allow time to establish patient preference with regard to escalation to IMV (grade D).

\section{End of life care}

Good practice points

- Although removal of the NIV mask may be agreed as preferable, a dignified and comfortable death is possible with it in place.

- Clinicians delivering NIV or IMV should have training in end of life care and the support of palliative care teams.

\section{Novel therapies \\ Extracorporeal $\mathrm{CO}_{2}$ removal $\left(\mathrm{ECCO}_{2} \mathrm{R}\right)$ \\ Recommendations}

81. If local expertise exists, $\mathrm{ECCO}_{2} \mathrm{R}$ might be considered:

- If, despite attempts to optimise IMV using lung protective strategies, severe hypercapnic acidosis $(\mathrm{pH}$ $<7.15)$ persists (grade D).

- When 'lung protective ventilation' is needed but hypercapnia is contraindicated for example, coexistent brain injury (grade D).

- For IMV patients awaiting a lung transplant (grade D).

Good practice point

$\mathrm{ECCO}_{2} \mathrm{R}$ is an experimental therapy and should only be used by specialist intensive care teams trained in its use, and where additional governance arrangements are in place, or in the setting of a research trial.

\section{Helium/oxygen ventilation}

\section{Recommendation}

82. Heliox should not be used routinely in the management of AHRF (grade B).

\section{CONCLUSION}

A central theme of the guideline is to promote integration in the planning and delivery of NIV and IMV in acute hypercapnic respiratory failure. Despite evidence demonstrating the value of non-invasive ventilation, its introduction into routine clinical practice has not delivered the expected patient benefit and it is likely that NIV provision has, inadvertently, reduced access to IMV in AECOPD and the other causes of AHRF in the UK. The widespread introduction of an adequately resourced and integrated AHRF patient pathway in our hospitals is strongly recommended. It is expected that this will lead to improved clinical outcomes and patient experience.

Author affiliations

${ }^{1}$ British Thoracic Society, London, UK

${ }^{2}$ British Thoracic Society, London, UK

${ }^{3}$ St James's University Hospital, Leeds, UK

${ }^{4}$ Barts Health NHS Trust, London, UK

${ }^{5}$ Department of Respiratory Research, University Hospitals of Coventry and Warwickshire NHS Trust, Coventry, UK

${ }^{6}$ Sheffield Teaching Hospitals NHS Foundation Trust, Sheffield, UK

${ }^{7}$ Department of Respiratory, Scottish Pulmonary Vascular Unit, Glasgow, UK

${ }^{8}$ Royal Surrey County Hospital NHS Foundation Trust and Faculty of Health and Medical Sciences, University of Surrey, Guildford, UK

${ }^{9}$ Academic Respiratory Unit, University of Bristol, Bristol, UK North Bristol

Lung Centre, North Bristol NHS Trust, Bristol, UK

${ }^{10}$ University Hospital of South Manchester NHS Foundation Trust,

Manchester, UK

${ }^{11}$ Emergency Department, Manchester Royal Infirmary, Central Manchester University Hospitals NHS Foundation Trust, Manchester, Manchester M13 9WL, UK

${ }^{12}$ University of Plymouth, Plymouth, UK

${ }^{13}$ Department of Physiotherapy, Guy's and St Thomas' NHS Foundation Trust, St Thomas' Hospital, London, UK

${ }^{14}$ Aintree University Hospital, Liverpool, UK

${ }^{15}$ CLAHRC, Imperial College, London, UK

${ }^{16}$ Queen's Medical Centre, Nottingham, UK

${ }^{17}$ Royal College of Physicians, London, UK

Acknowledgements The authors acknowledge assistance with developing the aims and structure of the guidelines provided by Martin Wildman, Simon Baudouin, Robert Winter, Sara Bolton, Mandy Odell, David Smith and the members of the BTS Standards of Care Committee.

Collaborators BTS Standards of Care Committee members: Colin Gelder, James Dodd and Caroline Patterson.

Contributors ACD chaired the guideline group, and led the drafting and revision of the document. He has final responsibility for the guideline. SB, $M E, D K, C G$ and $A G$ drafted and revised the paper. CC, BC-B, JD, TF, BF, LM, $\mathrm{LMcD}, \mathrm{RP}, \mathrm{CP}, \mathrm{MS}$ and $\mathrm{LT}$ conducted appraisal of the literature and provided draft sections of the document.

Representation Dr Bob Winter and Sara Bolton represented the Intensive Care Society, Dr Bernard Foëx represented the College of Emergency 
Medicine, Dr Daniel Kennedy represented the Royal College of Anaesthetists and Surgeon Captain Lynn Thomas represented the Royal College of Physicians.

Competing interests $A C D$ declares being paid as a consultant to Smith Medical between 2008 and 2013. ME declares he has received an honorarium, and travel and subsistence expenses for speaking at a meeting in Australia organised by Resmed, a Respiratory Sleep and Ventilation company. He has received an honorarium and travel expenses for speaking at a meeting in London organised by Phillips Respironics, a Respiratory Sleep and Ventilation company. He has received travel and subsistence expenses for speaking at a meeting in China organised by Curative Medical Inc, a Respiratory Sleep and Ventilation company. He has received travel expenses for speaking at meetings in India organised by Phillips Respironics, a Respiratory Sleep and Ventilation company. AG declares being paid as a consultant and receiving honoraria and travel expenses for speaking at meetings organised by Armstrong Medical Ltd in the UK, between 2014 and 2015.

Provenance and peer review Not commissioned; internally peer reviewed.

Data sharing statement No additional data are available.

Open Access This is an Open Access article distributed in accordance with the Creative Commons Attribution Non Commercial (CC BY-NC 4.0) license, which permits others to distribute, remix, adapt, build upon this work noncommercially, and license their derivative works on different terms, provided the original work is properly cited and the use is non-commercial. See: http:// creativecommons.org/licenses/by-nc/4.0/

\section{REFERENCES}

1. British Thoracic Society Standards of Care Committee. Non-invasive ventilation in acute respiratory failure. Thorax 2002;57:192-211.

2. Plant PK, Owen J, Elliott MW. One year period prevalence study of respiratory acidosis in acute exacerbation of COPD; implications for the provision of non- invasive ventilation and oxygen administration. Thorax 2000;55:550-4.

3. Davidson AC. Report British Thoracic Society NIV Audit 2011. https:// www.brit-thoracic.org.uk/document-library/audit-and-qualityimprovement/audit-reports/bts-adult-niv-audit-report-2011/

4. Davies M. Report British Thoracic Society NIV Audit 2012. https://www.brit-thoracic.org.uk/document-library/ audit-and-quality-improvement/audit-reports/ bts-adult-niv-audit-report-2012/

5. Davies M. Report British Thoracic Society NIV Audit 2013. https:// www.brit-thoracic.org.uk/document-library/audit-and-qualityimprovement/audit-reports/bts-adult-niv-audit-report-2013/

6. Davidson AC. Towards a comprehensive ventilatory strategy for acute exacerbations of COPD. JICS 2008;9:5-7.

7. Roberts CM, Stone RA, Buckingham RJ, et al. Acidosis, non-invasive ventilation and mortality in hospitalised COPD exacerbations. Thorax 2011;66:43-8.

8. Gupta D, Keogh B, Chung KF, et al. Characteristics and outcome for admissions to adult, general critical care units with acute severe asthma: a secondary analysis of the ICNARC Case Mix Programme Database. Crit Care 2004;8:R112-21.

9. British Thoracic Society/Intensive Care Society Guideline for the ventilatory management of acute hypercapnic respiratory failure in adults 2016. Thorax 71:ii1-ii35. doi:10.1136/thoraxjnl-2015-208209 